\title{
Postmastectomy radiation therapy can improve survival for breast cancer patients with 1-3 positive axillary lymph nodes: a retrospective cohort study using the SEER database
}

\author{
Jian Yang ${ }^{1 *}$, Xiao Zhang ${ }^{2 *}$, Yifeng $\mathrm{Ye}^{2}$, Yunyan $\mathrm{Lin}^{3}$, Qingmo Yang ${ }^{3}$, Haoyang $\mathrm{Cai}^{1}$ \\ ${ }^{1}$ Center of Growth, Metabolism, and Aging, Key Laboratory of Bio-Resources and Eco-Environment, College of Life Sciences, Sichuan University, \\ Chengdu, China; ${ }^{2}$ Department of Breast Surgery, Sichuan Provincial People's Hospital, University of Electronic Science and Technology of China, \\ Chengdu, China; ${ }^{3}$ Department of Breast Surgery, The First Affiliated Hospital of Xiamen University, Xiamen, China \\ Contributions: (I) Conception and design: Q Yang, H Cai; (II) Administrative support: Q Yang; (III) Provision of study materials or patients: X Zhang; \\ (IV) Collection and assembly of data: X Zhang, J Yang, Y Lin; (V) Data analysis and interpretation: X Zhang, J Yang, Y Lin; (VI) Manuscript writing: \\ All authors; (VII) Final approval of manuscript: All authors. \\ "These authors contributed equally to this work. \\ Correspondence to: Qingmo Yang, MD. Department of Breast Surgery, The First Affiliated Hospital of Xiamen University, 55 Zhenhai Road, Siming \\ District, Xiamen 361003, China. Email: yqm8383@163.com; Haoyang Cai, PhD. Center of Growth, Metabolism, and Aging, Key Laboratory of \\ Bio-Resources and Eco-Environment, College of Life Sciences, Sichuan University, No. 29 Jiuyanqiao Wangjiang Road, Chengdu 610064, China. \\ Email: haoyang.cai@scu.edu.cn.
}

Background: Postmastectomy radiation (PMRT) is an important adjuvant treatment for high-risk breast cancer. However, evidence concerning its efficacy in promoting survival of patients with 1-3 positive axillary lymph nodes remains insufficient.

Methods: We identified 57,793 patients, diagnosed from 2010-2015, from the Surveillance, Epidemiology, and End Results database, including 15,126 cases treated with beam radiation and 42,667 cases with none/ unknown radiation. A Kaplan-Meier curve was utilized to compare survival of the two groups. We used univariate and multivariate Cox proportional hazard models to identify independent prognostic factors presented as hazard ratios (HRs) and 95\% confidence intervals (CIs). For subgroup analysis, patients were stratified according to lymph node status, tumor size, and molecular subtypes.

Results: The PMRT group showed more aggressive clinicopathological features, including higher grades $(\mathrm{P}<0.001)$, larger tumor sizes $(\mathrm{P}<0.001)$, more lymph nodes $(\mathrm{P}<0.001)$, younger ages $(\mathrm{P}<0.001)$, more ERnegative cases $(\mathrm{P}<0.001)$, more $\mathrm{PR}$-negative cases $(\mathrm{P}<0.001)$, and more HER2 overexpression $(\mathrm{P}<0.001)$. In addition, the PMRT group received more radical surgeries $(\mathrm{P}<0.001)$ and more chemotherapy $(\mathrm{P}<0.001)$. In the multivariate Cox proportional hazard regression analysis, the PMRT group exhibited improved survival in terms of breast cancer specific survival (BCSS) (HR, 0.74; 95\% CI, 0.68-0.81; $\mathrm{P}<0.001$ ) and overall survival $(\mathrm{OS})(\mathrm{HR}, 0.72 ; 95 \% \mathrm{CI}, 0.67-0.78 ; \mathrm{P}<0.001)$. After stratification according to positive axillary lymph nodes, the PMRT group showed improved BCSS and OS in the LN 1 to 3 subgroup (HR, 0.74; 95\% CI, 0.64-0.85; $\mathrm{P}<0.001$ and HR, 0.68; 95\% CI, 0.60-0.78; $\mathrm{P}<0.001$, respectively). For patients with $1-3$ positive axillary lymph nodes and T1-2 tumors, the PMRT group still showed improved BCSS and OS (HR, 0.823; 95\% CI, 0.69-0.99; P=0.04 and HR, 0.75; 95\% CI, 0.64-0.88; $\mathrm{P}<0.001$, respectively). In the subgroup analysis, PMRT remained a significant favorable prognostic factor in T2 and HER2-/HR+ subtype $(\mathrm{P}<0.05)$. Conclusions: This study suggests that PMRT can confer a survival benefit to breast cancer patients with 1-3 positive axillary lymph nodes, even with modern treatment options. Furthermore, for patients with 1-3 positive axillary lymph nodes and T1-2 tumors, PMRT can still provide survival benefits. 
Keywords: Breast cancer; postmastectomy radiation; positive axillary lymph nodes; survival; molecular subtypes

Submitted Nov 28, 2020. Accepted for publication Mar 24, 2021.

doi: $10.21037 /$ tcr-20-3337

View this article at: http://dx.doi.org/10.21037/tcr-20-3337

\section{Introduction}

Postmastectomy radiation therapy (PMRT) can eliminate microscopic residual disease in the chest wall and regional lymph nodes, which may become sources of locoregional recurrence (LRR) and distant metastasis. According to some guidelines, PMRT has been recommended for patients with four or more positive axillary lymph nodes but not for patients with negative axillary lymph nodes (1-5). However, the effectiveness of PMRT for patients with 1-3 positive axillary lymph nodes remains controversial and was not firmly recommended by these guidelines. There have been some prospective clinical trials focusing on this patient group. The MA25 study was designed to randomize patients with 1-3 positive axillary lymph nodes to receive radiotherapy or not after mastectomy. Unfortunately, this trial was closed. The European SUPREMO trial was opened to assess the effect of PMRT on intermediaterisk patients. This trial randomized patients to local regional radiotherapy or no radiotherapy after mastectomy. However, it will take years before a definitive result is obtained (6).

Despite a lack of data from randomized controlled trials, there have been some retrospective studies regarding this question. Overgaard et al. (7) conducted a subgroup analysis of the DBCG $82 \mathrm{~b}$ and $\mathrm{c}$ randomized trials and only included 1,152 patients who had eight or more axillary lymph nodes removed. The 15 -year LRR was $10 \%$ and $51 \%$ for the PMRT group and no-PMRT group $(\mathrm{P}<0.001)$, respectively, for patients with four or more positive lymph nodes and $4 \%$ and $27 \%(\mathrm{P}<0.001)$ for patients with $1-3$ positive lymph nodes, respectively. Similarly, the PMRT group demonstrated significantly improved 15 -year OS for both patients with four or more positive lymph nodes $(21 \%$ vs. $12 \%, \mathrm{P}=0.03)$ and with $1-3$ positive lymph nodes $(57 \%$ vs. $48 \%, \mathrm{P}=0.03$ ). In 2014, McGale et al. (8) published a landmark meta-analysis of individual data including 8,135 patients from 22 trials between 1964 and 1986. For 700 patients without positive axillary lymph nodes, PMRT had no significant effect on LRR or breast cancer mortality. For 1,772 patients with four or more axillary lymph nodes,
PMRT reduced LRR (from $32.1 \%$ to $13.0 \%$ at 10 years, $\mathrm{P}<0.0001$ ), overall recurrence (from $75.1 \%$ to $66.3 \%$ at 10 years, $2 \mathrm{P}=0.0003$ ), and breast cancer mortality (from $80.0 \%$ to $70.7 \%$ at 20 years, $2 \mathrm{P}=0.04$ ). Notably, for 1,314 patients with 1-3 positive axillary lymph nodes, PMRT significantly reduced the LRR (from $20.3 \%$ to $3.8 \%$ at 10 years, $2 \mathrm{P}<0.00001$ ), overall recurrence (from $45.7 \%$ to $34.2 \%$ at 10 years, $2 \mathrm{P}=0.00006$ ), and breast cancer mortality (from $50.2 \%$ to $42.3 \%$ at 20 years, $2 \mathrm{P}=0.01$ ). Even for the 1,133 patients with $1-3$ positive axillary lymph nodes who received systemic therapy, PMRT significantly reduced LRR, overall recurrence, and breast cancer mortality. Due to the accumulating evidence in favor of PMRT for patients with 1-3 positive axillary lymph nodes, the National Comprehensive Cancer Network guidelines suggest that healthcare providers 'strongly consider radiation therapy to chest wall and infraclavicular region, supraclavicular area, internal mammary nodes, and any part of the axillary bed at risk' for this group of patients (evidence category IIA) (9).

However, these studies have some limitations. These trials were started decades ago. For example, the patients in the DBCG 82b and c trials were treated in the 1970s and 1980 s, and the patients included in the 2014 EBCTCG meta-analysis were treated between 1964 and 1986. Firstly, they do not reflect state-of-the-art treatments. Since that time, there have been many improvements in detection, surgical techniques, pathological reviews, and systemic therapy regimens. First, most patients received cyclophosphamide-methotrexate-fluorouracil (CMF) chemotherapy, but more intensive anthracycline and/ or taxane-based regimens are widely used in the present. Second, some studies applied no, or less intensive, endocrine therapy. For example, in the DBCG $82 \mathrm{~b}$ and c trials, endocrine therapy was given based on postmenopausal status rather than on ER positivity. Additionally, in the DBCG 82 c trial, postmenopausal patients were given $30 \mathrm{mg}$ tamoxifen daily for 1 year, whereas current patients will receive aromatase inhibitors for 5 years or longer. Third, for HER2-positive patients, contemporary therapy includes targeted therapy (e.g., Herceptin). Because these improvements in adjuvant therapy can reduce LRR and 
improve survival, PMRT may not be necessary anymore. However, radiation technology has improved a lot, which can improve patients' survival (10). Furthermore, these trials did not contain some important clinicopathological factors, such as ER and/or PR and HER2 statuses; hence, this may have introduced bias into the retrospective analyses.

Because it remains uncertain whether current breast cancer patients with 1-3 positive lymph nodes can benefit from PMRT, we performed a population-based study to compare the prognoses of breast cancer patients with 1-3 positive axillary lymph nodes with or without PMRT utilizing the Surveillance, Epidemiology, and End Results (SEER) database. We present the following article in accordance with the STROBE reporting checklist (available at http://dx.doi.org/10.21037/tcr-20-3337).

\section{Methods}

\section{Patients}

We signed the Data-Use Agreement for the Surveillance, Epidemiology, and End Results (SEER) Program Research Data (1973-2015) custom database (http://www.seer. cancer.gov). The SEER 18 database contains data from Atlanta, Connecticut, Detroit, Hawaii, Iowa, New Mexico, San Francisco-Oakland, Seattle-Puget Sound, Utah, Los Angeles, San Jose-Monterey, rural Georgia, and the Alaska Native Tumor Registry and the registries of greater California, Kentucky, Louisiana, New Jersey, and greater Georgia. SEER collects demographic and clinical information for patients and makes it available for analysis through custom databases by request. SEER*Stat (version 8.3.5) was used to download data from the SEER 18 custom database. This database contains data in the SEER 18 registries research database in addition to radiotherapy and chemotherapy information. We selected female breast cancer patients according to the following criteria: (I) patients were histologically confirmed as breast cancer; (II) the histologic subtype is invasive ductal cancer (according to ICD-O-3); (III) patients were diagnosed between 2010 and 2015; (IV) breast cancer is the patient's first and only tumor; (V) patients without metastases that received mastectomy. In total, 72,442 patients were primarily included. We excluded 2,447 cases for which radiotherapy had been recommended but for which it was unknown if it was administered and 158 cases of radiation for which the method or source was not specified. We excluded 6 cases of radioisotope use, 66 cases of radioactive implants, and 20 cases of combined beam and implants/isotopes. We excluded 366 cases of non-postsurgical radiotherapy, including beam and intraoperative radiation, radiation before and after surgery, radiation prior to surgery, surgery both before and after radiation, sequence unknown, intraoperative radiation with other radiation before/after surgery. We excluded 11,586 cases with missing data on one or more covariates. Finally, 57,793 patients were eligible for this study, including 15,126 cases of beam radiation and 42,667 cases of none/unknown radiation. The study was conducted in accordance with the Declaration of Helsinki (as revised in 2013). This article does not contain any studies with human participants or animals performed by any of the authors. Informed consent was not required because this was a population-based retrospective study, and no personal information was involved.

\section{Construction of variables}

All variables were extracted from SEER records. In this study, we included radiation (PRMT or no PMRT), race (white, black, or other), grade (I, II, III, or IV), tumor size (T1, T2, T3, or T4), lymph nodes $(0,1-3$, or 4 or more), diagnosis year (2010-2011, 2012-2013, or 2014-2015), age at diagnosis $(15-34,35-39,40-49,50-59,60-69,70-79$, or 80-100), ER status (positive or negative), PR status (positive or negative), HER2 status (positive or negative), surgery type (subcutaneous mastectomy and simple mastectomy, modified radical mastectomy and radical mastectomy, and extended radical mastectomy), and chemotherapy (yes or no/unknown). PMRT was defined as beam radiation, and no PMRT was defined as no/unknown radiation. ER- and PRborderline cases were classified as positive, while HER2borderline cases were classified as missing data. We focus on breast cancer specific survival (BCSS) and overall survival (OS). BCSS was defined as the time from diagnosis to death from breast cancer, while OS was defined as the time from diagnosis to death due to any cause. Loss of follow-ups were censored.

\section{Statistical analysis}

We used the Chi-squared test to compare the baseline characteristics of the two groups. The Kaplan-Meier method and log-rank test were performed to compare the survival distribution between the two groups. Additionally, the competing risk model was performed to compare the distribution of BCSS between two groups. The univariate and multivariate Cox proportional hazard regression models 
were utilized to calculate the hazard ratios (HRs) and 95\% confidence intervals (CIs). Subgroup analysis was used to compare the distribution of survival between the two groups after stratification by different covariates. In each subgroup, the multivariate Cox proportional hazard regression model was utilized to calculate the HR and $95 \%$ CI. All subgroup analyses were presented with forest plots.

We performed all statistical analyses with $\mathrm{R}$ (version 3.2.4, R project for Statistical Computing, Austria, https:// www.R-project.org/). All statistical analyses were two-sided, and $\mathrm{P}<0.05$ was defined as statistically significant.

\section{Results}

\section{Baseline patient characteristics}

Table 1 shows the demographic and clinicopathological characteristics of the patients. The follow-up time ranged from 0 to 71 months, with a median of 31 months. There was a total of 4,288 deaths, 2,905 that were caused by breast cancer during follow-up. The two groups had significantly different distributions of clinicopathological characteristics. The tumors in the PMRT group were more aggressive. Specifically, the PMRT group exhibited tumors of higher grade (III and IV, $55.4 \%$ vs. $41.0 \%, \mathrm{P}<0.001$ ), larger tumor sizes (T2, 3 , and $4,76.5 \%$ vs. $42.9 \%, \mathrm{P}<0.001$ ), more lymph-node involvement (LN-positive, $78.6 \%$ vs. 29.2\%, $\mathrm{P}<0.001)$, more ER-negative (26.2\% vs. $21.6 \%, \mathrm{P}<0.001)$ and PR-negative cases $(37.4 \%$ vs. $32.1 \%, \mathrm{P}<0.001)$, more HER2 overexpression $(23.8 \%$ vs. $20.5 \%, \mathrm{P}<0.001)$, and younger ages $(15-59,68.5 \%$ vs. $56.5 \%, \mathrm{P}<0.001)$. In addition, the PMRT group received more radical surgeries (MRM, RM, and ERM, $61.3 \%$ vs. $30.9 \%, \mathrm{P}<0.001$ ) and more chemotherapy $(87.2 \%$ vs. $44.4 \%, \mathrm{P}<0.001)$.

\section{Comparison of survival between the PMRT and no-PMRT groups}

We performed Kaplan-Meier analysis to compare survival between the PMRT and no-PMRT groups. The PMRT group had a significantly lower survival probability compared with the no-PMRT group in both BCSS (HR, 1.98; 95\% CI, 1.84-2.13; $\mathrm{P}<0.001)$ and OS (HR, 1.38; 95\% CI, 1.29-1.47; $\mathrm{P}<0.001$ ) (Figure 1). The five-year BCSS rates were $85.2 \%$ (95\% CI, 84.3-86.1\%) and $92.4 \%$ (95\% CI, 92.0-92.8\%) in the PMRT and no-PMRT groups, respectively. Similarly, the OS rates were $82.7 \%$ (95\% CI, $81.7-83.6 \%$ ) and $87.5 \%$ (95\% CI, $87.0-88.0 \%)$ in the two groups, respectively. In the competing risk model, the PMRT group had higher probability of cumulative incidence compared with no PRMT group when considering death caused by breast cancer (Figure S1).

In the univariate model, radiation, race, grade, tumor size, lymph-node status, diagnosis year (2012-2013), age group (40-49, 50-59, 60-69, and 80-99), ER status, PR status, HER2 status, surgery, and chemotherapy were significantly associated with BCSS (Table 2). In the multivariate model, radiation, race, grade, tumor size, lymph-node status, age group (70-79 and 80-99), ER status, PR status, HER2 status, surgery, and chemotherapy remained significantly related to BCSS after controlling for the above factors. In multivariable analysis, the PMRT group had a higher risk of BCSS (HR, 0.74; 95\% CI, 0.68$0.81 ; \mathrm{P}<0.001)$. Several characteristics, including radiation, race, tumor size, lymph-node status, diagnosis year (20142015), age group (40-49, 70-79, and 80-99), ER status, PR status, HER2 status and surgery were significantly related to OS in the univariate model (Table 3). In the multivariate model, radiation, race, grade, tumor size, lymph-node status, age group (60-69, 70-79, and 80-99), ER status, PR status, HER2 status, surgery, and chemotherapy remained significantly related to OS. It is noteworthy that PMRT was a significantly better prognostic predictor for OS (HR, 0.72; 95\% CI, 0.67-0.78; $\mathrm{P}<0.001)$.

\section{Comparison of survival between the PMRT and no-PMRT groups based on LN subgroups}

We stratified all patients into three subgroups based on positive axillary lymph nodes. As presented in Figures 2 and 3, the PMRT group showed improved BCSS and OS in the LN 1 to 3 subgroup (HR, 0.74; 95\% CI, 0.64-0.85; $\mathrm{P}<0.001$ and HR, 0.68; 95\% CI, 0.60-0.78; $\mathrm{P}<0.001$, respectively) and $\mathrm{LN} \geq 4$ subgroup (HR, 0.64; 95\% CI, 0.56-0.72; $\mathrm{P}<0.001$ and HR, 0.62; 95\% CI, 0.56-0.70; $\mathrm{P}<0.001$, respectively) but not in the LN 0 subgroup (HR, 1.14; 95\% CI, $0.93-$ 1.42; $\mathrm{P}=0.211$ and $\mathrm{HR}, 1.04 ; 95 \% \mathrm{CI}, 0.87-1.23 ; \mathrm{P}=0.704$, respectively).

\section{Comparison of survival between the PMRT and no-PMRT groups in the LN 1-3 and T1-2 subgroup}

In the univariate model, PMRT, grade, race, age group (80-99), ER status, PR status, HER2 status, surgery, and chemotherapy were significantly related to BCSS (Table 4). After controlling for the above factors, beam radiation, 
Table 1 Patient characteristics

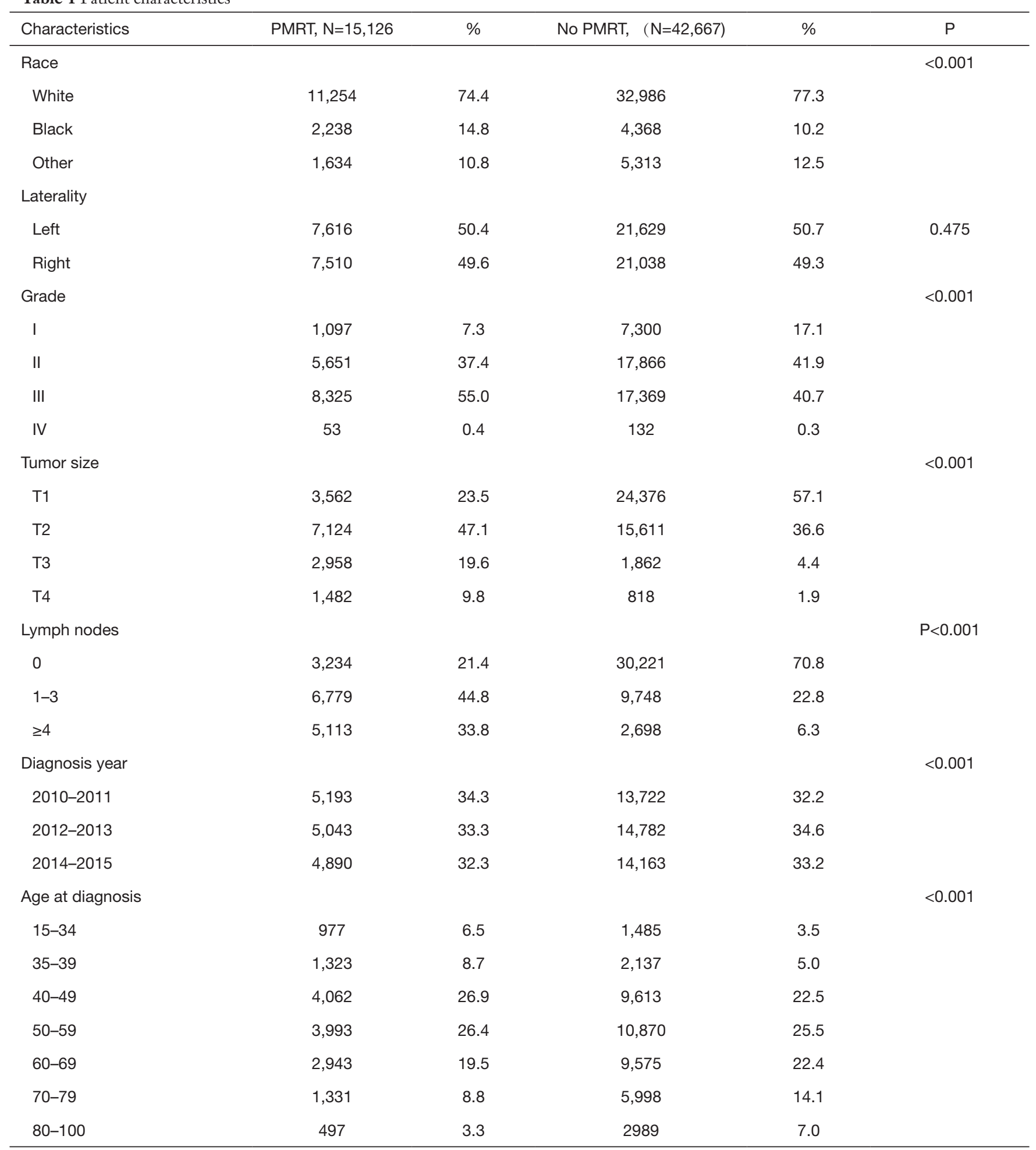

Table 1 (continued) 
Table 1 (continued)

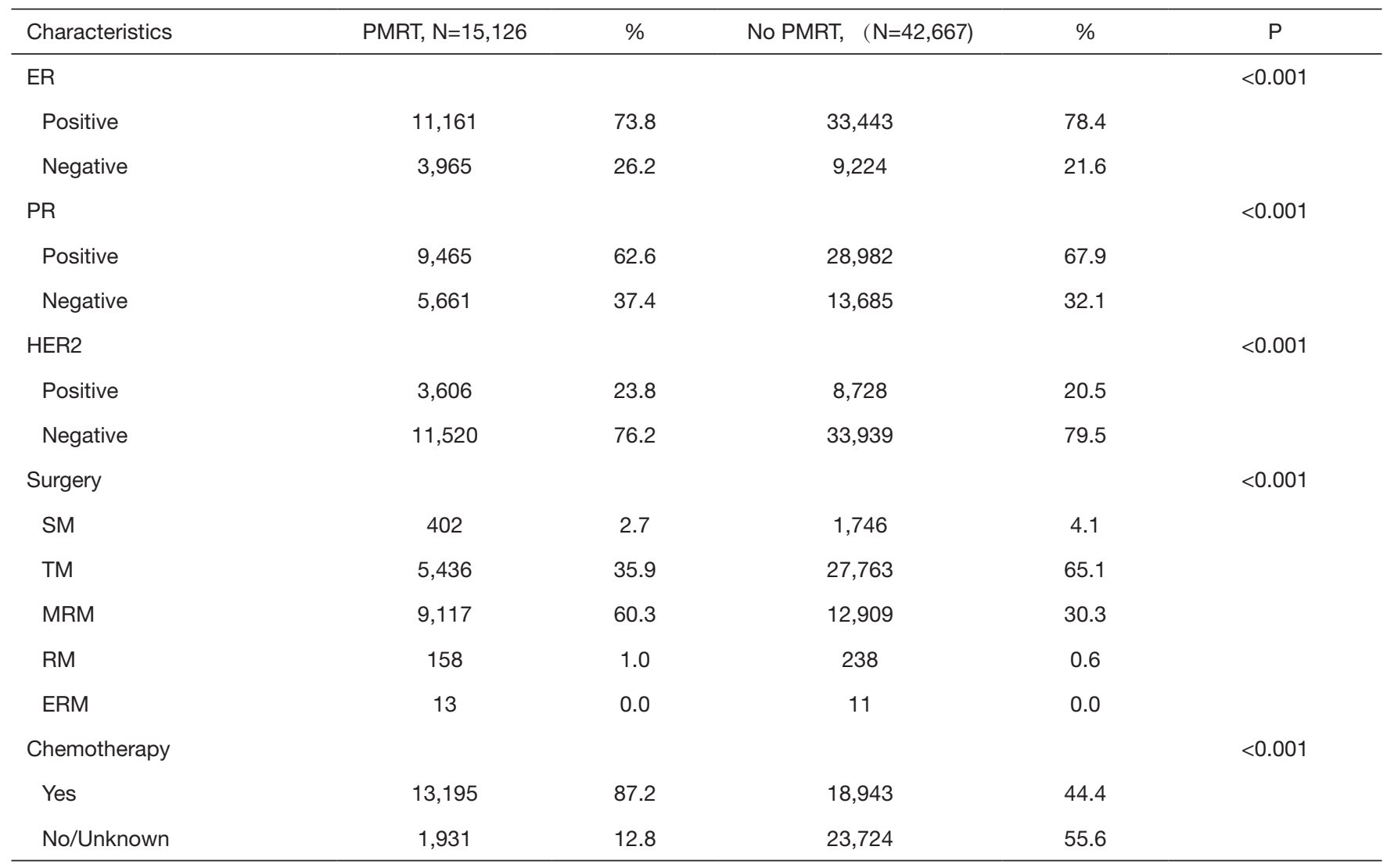

ER, estrogen receptor; PR, progesterone receptor; HER2, human epidermal growth factor receptor 2; SM, Subcutaneous mastectomy; TM, total mastectomy; MRM, modified radical mastectomy; RM, radical mastectomy; ERM, extended radical mastectomy.

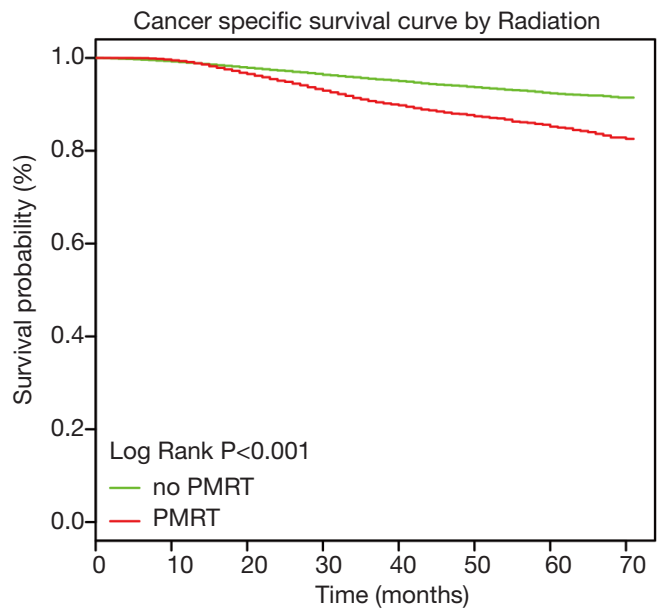

Number at risk by time (survival probability\%)

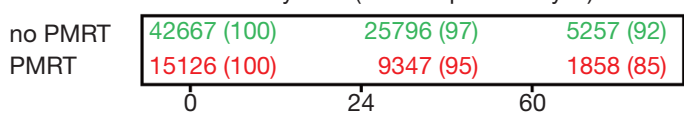

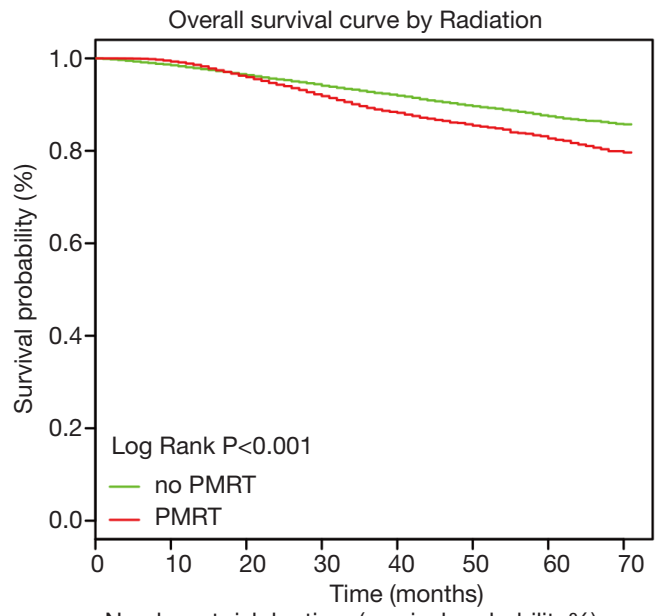

Number at risk by time (survival probability\%)

\begin{tabular}{l|rrr|} 
no PMRT & $42667(100)$ & $25796(95)$ & $5257(87)$ \\
PMRT & $15126(100)$ & $9347(94)$ & $1858(85)$ \\
\hline & 0 & 24 & 60
\end{tabular}

Figure 1 Kaplan-Meier curves of breast cancer-specific survival (BCSS) and overall survival (OS) based on radiation for all patients, PMRT vs. no PMRT. 
Table 2 Univariate and multivariate Cox proportional hazard model of breast cancer-specific survival (BCSS)

\begin{tabular}{|c|c|c|c|c|c|c|}
\hline Variables & \multicolumn{3}{|c|}{ Univariate analysis } & \multicolumn{3}{|c|}{ Multivariate analysis } \\
\hline \multicolumn{7}{|l|}{ Radiation } \\
\hline No PMRT & & ref & & & ref & \\
\hline PMRT & 1.98 & $1.84,2.13$ & 0.000 & 0.74 & $0.68,0.81$ & 0.000 \\
\hline White & & ref & & & ref & \\
\hline Black & 1.75 & $1.60,1.93$ & 0.000 & 1.25 & $1.14,1.37$ & 0.000 \\
\hline Other & 0.59 & $0.51,0.68$ & 0.000 & 0.66 & $0.57,0.77$ & 0.000 \\
\hline \multicolumn{7}{|l|}{ Grade } \\
\hline \multicolumn{7}{|l|}{ Tumor Size } \\
\hline $\mathrm{T} 1$ & & ref & & & ref & \\
\hline $\mathrm{T} 2$ & 3.79 & $3.41,4.21$ & 0.000 & 2.13 & $1.91,2.38$ & 0.000 \\
\hline T3 & 8.83 & $7.82,9.96$ & 0.000 & 3.98 & $3.50,4.54$ & 0.000 \\
\hline $\mathrm{T} 4$ & 14.75 & $12.97,16.77$ & 0.000 & 5.38 & $4.67,6.19$ & 0.000 \\
\hline \multicolumn{7}{|l|}{ Lymph nodes } \\
\hline 0 & & ref & & & ref & \\
\hline $1-3$ & 2.65 & $2.41,2.92$ & 0.000 & 2.33 & $2.10,2.59$ & 0.000 \\
\hline \multicolumn{7}{|l|}{ Age group } \\
\hline $15-34$ & & ref & & & ref & \\
\hline $35-39$ & 0.94 & $0.75,1.17$ & 0.557 & 1.01 & $0.81,1.26$ & 0.933 \\
\hline $40-49$ & 0.69 & $0.57,0.83$ & 0.000 & 0.86 & $0.71,1.04$ & 0.120 \\
\hline $50-59$ & 0.79 & $0.66,0.95$ & 0.013 & 0.93 & $0.78,1.12$ & 0.457 \\
\hline $60-69$ & 0.77 & $0.64,0.93$ & 0.006 & 1.01 & $0.84,1.22$ & 0.934 \\
\hline $70-79$ & 1.09 & $0.90,1.32$ & 0.376 & 1.38 & $1.14,1.68$ & 0.001 \\
\hline 80-99 & 2.43 & $2.00,2.94$ & 0.000 & 2.42 & $1.97,2.97$ & 0.000 \\
\hline \multicolumn{7}{|l|}{ ER } \\
\hline Positive & & ref & & & ref & \\
\hline Negative & 3.55 & $3.30,3.81$ & 0.000 & 1.59 & $1.43,1.76$ & 0.000 \\
\hline
\end{tabular}

Table 2 (continued) 
Table 2 (continued)

\begin{tabular}{|c|c|c|c|c|c|c|}
\hline Variables & \multicolumn{3}{|c|}{ Univariate analysis } & \multicolumn{3}{|c|}{ Multivariate analysis } \\
\hline \multicolumn{7}{|l|}{ PR } \\
\hline Positive & & ref & & & ref & \\
\hline Negative & 3.76 & $3.48,4.05$ & 0.000 & 2.17 & $1.95,2.41$ & 0.000 \\
\hline Positive & & ref & & & ref & \\
\hline Negative & 1.16 & $1.06,1.28$ & 0.002 & 1.82 & $1.65,2.00$ & 0.000 \\
\hline \multicolumn{7}{|l|}{ Surgery } \\
\hline SM and TM & & ref & & & ref & \\
\hline No/Unknown & & ref & & & ref & \\
\hline Yes & 1.73 & $1.60,1.88$ & 0.000 & 0.82 & $0.74,0.91$ & 0.000 \\
\hline
\end{tabular}

$\mathrm{HR}$, hazard ratio; $\mathrm{Cl}$, confidence interval; ER, estrogen receptor; PR, progesterone receptor; HER2, human epidermal growth factor receptor 2; SM, Subcutaneous mastectomy; TM, total mastectomy; MRM, modified radical mastectomy; RM, radical mastectomy; ERM, extended radical mastectomy. Multivariate analysis include radiation, race, grade, tumor size, lymph nodes, diagnosis year, age at diagnosis, ER status, PR status, HER2 status, surgery and chemotherapy.

race (other race), grade, age group (80-99), ER status, PR status, HER2 status, and surgery remained significantly related to BCSS in the multivariate model. The PMRT group had better BCSS (HR, 0.83; 95\% CI, 0.69-0.99; $\mathrm{P}=0.040)$ than the no-PMRT group. Some characteristics, including radiation, race, grade, age group (60-69, 70-79, and 80-99), ER status, PR status, HER2 status, surgery, and chemotherapy were significantly related to OS in the univariate model, as shown in Table 5. In the multivariate model, radiation, race (other race), grade, age group (70-79 and 80-99), ER status, PR status, HER2 status, surgery, and chemotherapy remained significantly related to OS. Specifically, PMRT was a significantly better prognostic predictor for OS (HR, 0.75; 95\% CI, 0.64-0.88; $\mathrm{P}<0.001$ ).

\section{Subgroup analysis of patients with 1-3 lymph nodes and T1-2 based on T stage and molecular subtype}

Because the effect of PMRT on survival may differ for different $T$ stages and molecular subtypes, subgroup analyses of survival were performed by stratifying $\mathrm{T}$ stage and molecular subtype. When stratified by T stage, PMRT can significantly improve BCSS at stages T2 (HR, 0.77;
95\% CI, 0.63-0.95; $\mathrm{P}=0.014)$ and $\mathrm{T} 4$ (HR, 0.61; 95\% CI, 0.42-0.88; $\mathrm{P}=0.009)$ but not at stages T1 (HR, 0.84; $95 \%$ CI, 0.57-1.25; $\mathrm{P}=0.395$ ) and T3 (HR, 0.79; 95\% CI, 0.581.07; $\mathrm{P}=0.127$ ) (Figure 4). PMRT can significantly improve OS at stages T2 (HR, 0.71; 95\% CI, 0.59-0.85; $\mathrm{P}<0.001$ ), T3 (HR, 0.74; 95\% CI, 0.56-0.97; $\mathrm{P}=0.029$ ), and T4 (HR, $0.55 ; 95 \% \mathrm{CI}, 0.39-0.77 ; \mathrm{P}=0.001$ ) but not at stage T1 (HR, 0.74; 95\% CI, 0.54-1.03; $\mathrm{P}=0.078$ ) (Figure 5).

As presented in Figures 6 and 7, the PMRT group showed improved BCSS and OS for the HER2-/HR+ subtype (HR, 0.72; 95\% CI, 0.55-0.95; $\mathrm{P}=0.021$ and HR, 0.65 ; 95\% CI, 0.52-0.81; $\mathrm{P}<0.001$, respectively) but no significant difference for the HER2+/HR+ (HR, 1.25; 95\% CI, 0.67-2.33; $\mathrm{P}=0.477$ and HR, 0.97; 95\% CI, 0.57-1.65; $\mathrm{P}=0.912$, respectively), HER2+/HR- (HR, 0.83; 95\% CI, $0.42-1.66 ; \mathrm{P}=0.600$ and HR, 0.65; $95 \%$ CI, 0.34-1.23; $\mathrm{P}=0.188$, respectively) and triple-negative (HR, 0.86; $95 \%$ CI, 0.65-1.15; $\mathrm{P}=0.320$ and HR, 0.84; 95\% CI, 0.65-1.10; $\mathrm{P}=0.209$, respectively) subtypes.

\section{Discussion}

We found that the PMRT group showed improved survival 
Table 3 Univariate and multivariate Cox proportional hazard model of overall survival

\begin{tabular}{|c|c|c|c|c|c|c|}
\hline Variables & \multicolumn{3}{|c|}{ Univariate analysis } & \multicolumn{3}{|c|}{ Multivariate analysis } \\
\hline \multicolumn{7}{|l|}{ Radiation } \\
\hline No PMRT & & ref & & & ref & \\
\hline PMRT & 1.38 & $1.29,1.47$ & 0.000 & 0.72 & $0.67,0.78$ & 0.000 \\
\hline White & & ref & & & ref & \\
\hline Black & 1.54 & $1.42,1.67$ & 0.000 & 1.27 & $1.17,1.37$ & 0.000 \\
\hline Other & 0.55 & $0.49,0.63$ & 0.000 & 0.64 & $0.56,0.72$ & 0.000 \\
\hline \multicolumn{7}{|l|}{ Grade } \\
\hline \multicolumn{7}{|l|}{ Tumor Size } \\
\hline $\mathrm{T} 1$ & & ref & & & ref & \\
\hline T2 & 2.59 & $2.40,2.80$ & 0.000 & 1.79 & $1.65,1.94$ & 0.000 \\
\hline T3 & 4.92 & $4.47,5.41$ & 0.000 & 3.03 & $2.73,3.36$ & 0.000 \\
\hline $\mathrm{T} 4$ & 8.33 & $7.52,9.24$ & 0.000 & 3.94 & $3.51,4.41$ & 0.000 \\
\hline \multicolumn{7}{|l|}{ Lymph nodes } \\
\hline 0 & & ref & & & ref & \\
\hline $1-3$ & 1.90 & $1.76,2.04$ & 0.000 & 1.77 & $1.63,1.92$ & 0.000 \\
\hline 2014-2015 & 0.88 & $0.77,01.00$ & 0.045 & 1.00 & $0.88,1.14$ & 0.946 \\
\hline \multicolumn{7}{|l|}{ Age group } \\
\hline $15-34$ & & ref & & & ref & \\
\hline 35-39 & 0.91 & $0.73,1.13$ & 0.394 & 0.97 & $0.78,1.20$ & 0.772 \\
\hline $40-49$ & 0.72 & $0.60,0.86$ & 0.000 & 0.85 & $0.71,1.02$ & 0.083 \\
\hline $50-59$ & 0.89 & $0.75,1.06$ & 0.187 & 0.99 & $0.83,1.18$ & 0.927 \\
\hline $60-69$ & 1.08 & $0.90,1.28$ & 0.403 & 1.27 & $1.07,1.52$ & 0.008 \\
\hline $70-79$ & 1.86 & $1.56,2.22$ & 0.000 & 2.04 & $1.71,2.44$ & 0.000 \\
\hline 80-99 & 5.12 & $4.30,6.09$ & 0.000 & 4.28 & $3.56,5.14$ & 0.000 \\
\hline \multicolumn{7}{|l|}{ ER } \\
\hline Positive & & ref & & & ref & \\
\hline Negative & 2.58 & $2.43,2.75$ & 0.000 & 1.53 & $1.41,1.67$ & 0.000 \\
\hline
\end{tabular}

Table 3 (continued) 
Table 3 (continued)

\begin{tabular}{|c|c|c|c|c|c|c|}
\hline Variables & \multicolumn{3}{|c|}{ Univariate analysis } & \multicolumn{3}{|c|}{ Multivariate analysis } \\
\hline \multicolumn{7}{|l|}{ PR } \\
\hline Positive & & ref & & & ref & \\
\hline Negative & 2.61 & $2.46,2.77$ & 0.000 & 1.73 & $1.59,1.89$ & 0.000 \\
\hline \multicolumn{7}{|l|}{ HER2 } \\
\hline Positive & & ref & & & ref & \\
\hline Negative & 1.23 & $1.14,1.33$ & 0.000 & 1.63 & $1.51,1.77$ & 0.000 \\
\hline \multicolumn{7}{|l|}{ Surgery } \\
\hline SM and TM & & ref & & & ref & \\
\hline No/Unknown & & ref & & & ref & \\
\hline Yes & 0.96 & $0.90,1.02$ & 0.162 & 0.69 & $0.64,0.75$ & 0.000 \\
\hline
\end{tabular}

$\mathrm{HR}$, hazard ratio; $\mathrm{Cl}$, confidence interval; ER, estrogen receptor; PR, progesterone receptor; HER2, human epidermal growth factor receptor 2; SM, Subcutaneous mastectomy; TM, total mastectomy; MRM, modified radical mastectomy; RM, radical mastectomy; ERM, extended radical mastectomy. Multivariate analysis include radiation, race, grade, tumor size, lymph nodes, diagnosis year, age at diagnosis, ER status, PR status, HER2 status, surgery and chemotherapy.

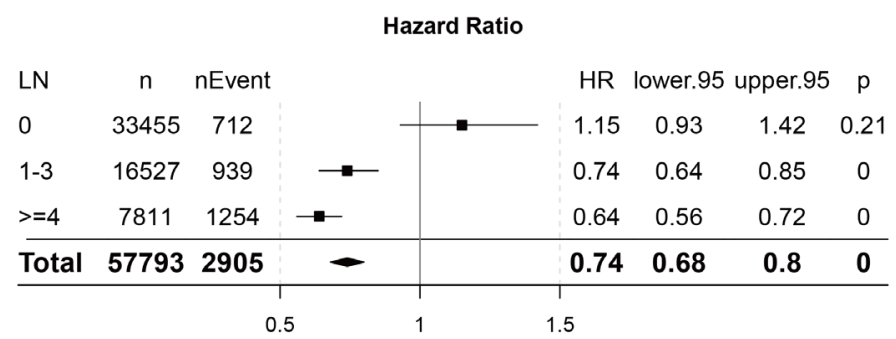

Figure 2 Forest plot of BCSS based on lymph nodes. The hazard ratio (HR) was calculated by multivariate Cox proportional hazard model adjusted for radiation, race, tumor size, diagnosis year, age group, ER status, PR status, HER2 status, surgery and chemotherapy. LN, lymph node. The data source was the whole patients.

Hazard Ratio

\begin{tabular}{|c|c|c|c|c|c|c|c|}
\hline LN & $\mathrm{n}$ & nEvent & & HR & lower.9 & pper. & $p$ \\
\hline 0 & 33455 & 1435 & & 1.03 & 0.87 & 1.23 & 0.7 \\
\hline $1-3$ & 16527 & 1352 & $\rightarrow$ & 0.68 & 0.6 & 0.78 & 0 \\
\hline$>=4$ & 7811 & 1501 & $\rightarrow-$ & 0.62 & 0.56 & 0.7 & 0 \\
\hline Total & 57793 & 4288 & - & 0.72 & 0.67 & 0.78 & 0 \\
\hline
\end{tabular}

Figure 3 Forest plot of OS based on lymph nodes. The hazard ratio (HR) was calculated by multivariate Cox proportional hazard model 
Table 4 Univariate and multivariate Cox proportional hazard model of breast cancer-specific survival (BCSS) in the LN 1-3 and T1,2 subgroup

\begin{tabular}{|c|c|c|c|c|c|c|}
\hline \multirow{2}{*}{ Variables } & \multicolumn{3}{|c|}{ Univariate analysis } & \multicolumn{3}{|c|}{ Multivariate analysis } \\
\hline & $\mathrm{HR}$ & $(95 \% \mathrm{Cl})$ & $P$ value & $\mathrm{HR}$ & $(95 \% \mathrm{Cl})$ & $P$ value \\
\hline \multicolumn{7}{|l|}{ Radiation } \\
\hline PMRT & 0.8 & $0.69,0.98$ & 0.025 & 0.83 & $0.69,0.99$ & 0.040 \\
\hline \multicolumn{7}{|l|}{ Race } \\
\hline Black & 1.36 & $1.10,1.69$ & 0.005 & 1.12 & $0.90,1.39$ & 0.325 \\
\hline Other & 0.53 & $0.38,0.75$ & 0.000 & 0.56 & $0.40,0.79$ & 0.001 \\
\hline \multicolumn{7}{|l|}{ Grade } \\
\hline I and II & & ref & & & ref & \\
\hline 2012-2013 & 0.92 & $0.76,1.11$ & 0.367 & 0.98 & $0.81,1.18$ & 0.806 \\
\hline 2014-2015 & 0.94 & $0.64,1.36$ & 0.729 & 1.00 & $0.69,1.46$ & 0.982 \\
\hline \multicolumn{7}{|l|}{ Age group } \\
\hline $15-34$ & & ref & & & ref & \\
\hline $35-39$ & 0.90 & $0.56,1.45$ & 0.665 & 0.84 & $0.52,1.36$ & 0.478 \\
\hline $40-49$ & 0.67 & $0.44,1.00$ & 0.050 & 0.75 & $0.50,1.12$ & 0.163 \\
\hline $50-59$ & 0.84 & $0.57,1.25$ & 0.400 & 0.87 & $0.58,1.29$ & 0.485 \\
\hline $60-69$ & 0.91 & $0.61,1.35$ & 0.625 & 1.01 & $0.67,1.52$ & 0.955 \\
\hline Positive & & ref & & & ref & \\
\hline Negative & 3.86 & $3.27,4.54$ & 0.000 & 2.20 & $1.75,2.78$ & 0.000 \\
\hline \multicolumn{7}{|l|}{ HER2 } \\
\hline Positive & & ref & & & ref & \\
\hline Negative & 1.36 & $1.09,1.68$ & 0.006 & 2.00 & $1.60,2.49$ & 0.000 \\
\hline \multicolumn{7}{|l|}{ Surgery } \\
\hline SM and TM & & ref & & & ref & \\
\hline MRM, RM and ERM & 1.38 & $1.17,1.64$ & 0.000 & 1.21 & $1.02,1.44$ & 0.026 \\
\hline \multicolumn{7}{|l|}{ Chemotherapy } \\
\hline No/Unknown & & ref & & & ref & \\
\hline Yes & 0.79 & $0.67,0.93$ & 0.006 & 0.88 & $0.71,1.09$ & 0.240 \\
\hline
\end{tabular}

HR, hazard ratio; Cl, confidence interval; PMRT, postmastectomy radiation therapy; ER, estrogen receptor; PR, progesterone receptor; HER2, human epidermal growth factor receptor 2; SM, Subcutaneous mastectomy; TM, total mastectomy; MRM, modified radical mastectomy; RM, radical mastectomy; ERM, extended radical mastectomy. Multivariate analysis include radiation, race, grade, diagnosis year, age at diagnosis, ER status, PR status, HER2 status, surgery and chemotherapy., 
Table 5 Univariate and multivariate Cox proportional hazard model of overall survival (OS) in the LN 1-3 and T1,2 subgroup

\begin{tabular}{|c|c|c|c|c|c|c|}
\hline \multirow{2}{*}{ Variables } & \multicolumn{3}{|c|}{ Univariate analysis } & \multicolumn{3}{|c|}{ Multivariate analysis } \\
\hline & $\mathrm{HR}$ & $(95 \% \mathrm{Cl})$ & $P$ value & $\mathrm{HR}$ & $(95 \% \mathrm{Cl})$ & $P$ value \\
\hline \multicolumn{7}{|l|}{ Radiation } \\
\hline PMRT & 0.60 & $0.52,0.70$ & 0.000 & 0.75 & $0.64,0.88$ & 0.000 \\
\hline \multicolumn{7}{|l|}{ Race } \\
\hline Black & 1.31 & $1.10,1.56$ & 0.003 & 1.19 & $1.00,1.43$ & 0.052 \\
\hline Other & 0.53 & $0.40,0.70$ & 0.000 & 0.59 & $0.45,0.78$ & 0.000 \\
\hline \multicolumn{7}{|l|}{ Grade } \\
\hline I and II & & ref & & & ref & \\
\hline 2012-2013 & 0.95 & $0.82,1.10$ & 0.479 & 1.00 & $0.87,1.17$ & 0.959 \\
\hline 2014-2015 & 0.85 & $0.64,1.13$ & 0.258 & 0.91 & $0.68,1.22$ & 0.526 \\
\hline \multicolumn{7}{|l|}{ Age group } \\
\hline $15-34$ & & ref & & & ref & \\
\hline $35-39$ & 0.87 & $0.54,1.40$ & 0.564 & 0.83 & $0.51,1.33$ & 0.432 \\
\hline $40-49$ & 0.74 & $0.50,1.09$ & 0.130 & 0.78 & $0.53,1.16$ & 0.216 \\
\hline $50-59$ & 1.04 & $0.71,1.52$ & 0.847 & 1.00 & $0.68,1.47$ & 0.994 \\
\hline $60-69$ & 1.47 & $1.00,2.14$ & 0.048 & 1.45 & $0.99,2.13$ & 0.056 \\
\hline Positive & & ref & & & ref & \\
\hline Negative & 2.54 & $2.24,2.89$ & 0.000 & 1.77 & $1.47,2.13$ & 0.000 \\
\hline \multicolumn{7}{|l|}{ HER2 } \\
\hline Positive & & ref & & & ref & \\
\hline Negative & 1.40 & $1.17,1.66$ & 0.000 & 1.76 & $1.47,2.10$ & 0.000 \\
\hline \multicolumn{7}{|l|}{ Surgery } \\
\hline SM and TM & & ref & & & ref & \\
\hline MRM, RM and ERM & 1.37 & $1.19,1.56$ & 0.000 & 1.23 & $1.07,1.40$ & 0.003 \\
\hline \multicolumn{7}{|l|}{ Chemotherapy } \\
\hline No/Unknown & & ref & & & ref & \\
\hline Yes & 0.47 & $0.42,0.54$ & 0.000 & 0.74 & $0.63,0.87$ & 0.000 \\
\hline
\end{tabular}

$\mathrm{HR}$, hazard ratio; $\mathrm{Cl}$, confidence interval; PMRT, postmastectomy radiation therapy; ER, estrogen receptor; PR, progesterone receptor; HER2, human epidermal growth factor receptor 2; SM, subcutaneous mastectomy; TM, total mastectomy; MRM, modified radical mastectomy; RM, radical mastectomy; ERM, extended radical mastectomy. Multivariate analysis include radiation, race, grade, diagnosis year, age at diagnosis, ER status, PR status, HER2 status, surgery and chemotherapy. 


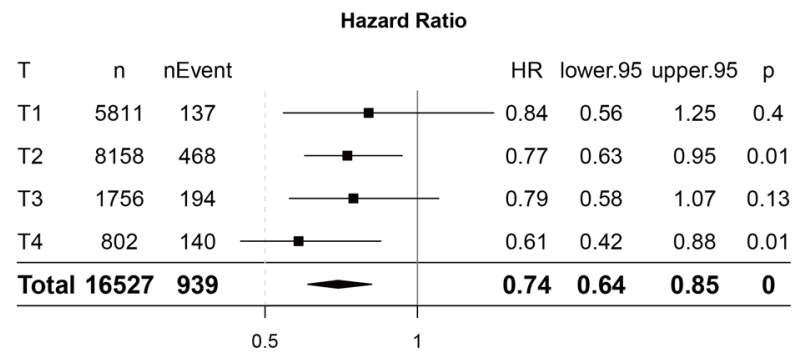

Figure 4 Forest plot of BCSS based on T stage. The hazard ratio (HR) was calculated by multivariate Cox proportional hazard model adjusted for radiation, race, diagnosis year, age group, ER status, PR status, HER2 status, surgery and chemotherapy. LN, lymph node. The data source was the LN1-3 subgroup.

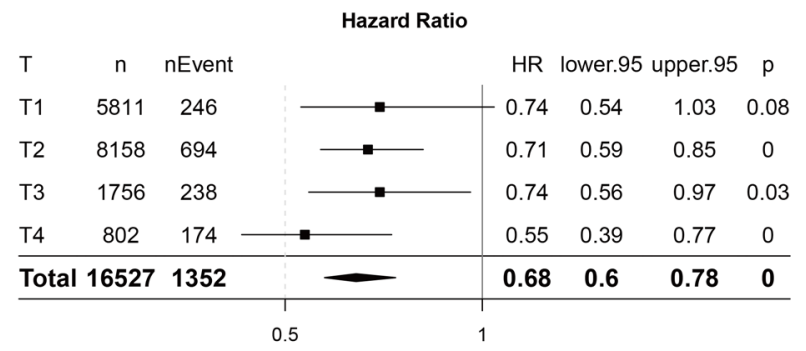

Figure 5 Forest plot of OS based on T stage. The hazard ratio (HR) was calculated by multivariate Cox proportional hazard model adjusted for radiation, race, diagnosis year, age group, ER status, PR status, HER2 status, surgery and chemotherapy. LN, lymph node. The data source was the LN1-3 subgroup.

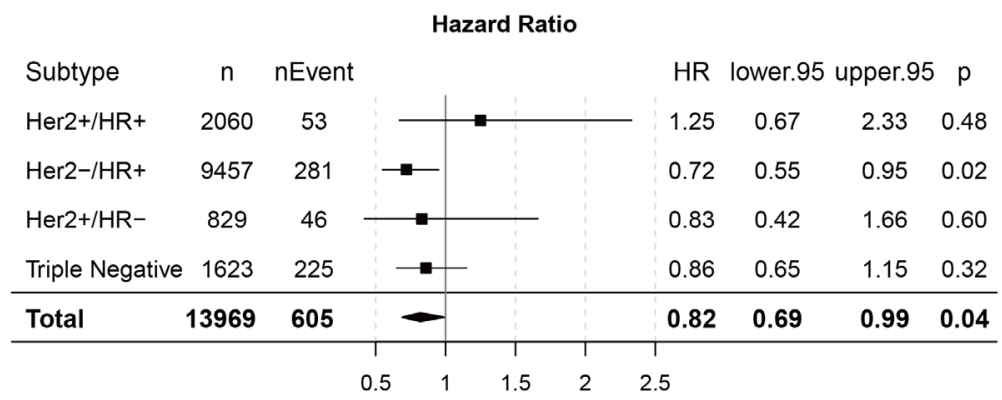

Figure 6 Forest plot of BCSS based on molecular subtypes. The hazard ratio (HR) was calculated by multivariate Cox proportional hazard model adjusted for radiation, race, diagnosis year, age group, surgery and chemotherapy. LN, lymph node. The data source was the LN1-3 and $\mathrm{T} 1,2$ subgroup.

in terms of BCSS after controlling for confounding variables. For patients with 1-3 positive axillary lymph nodes, PMRT improved both BCSS and OS. Among these patients, those with T1-2 tumors or T2 tumors of the HER2-/HR+ subtype also benefited from PMRT.

There have been many studies to determine whether
PMRT improves the survival of high-risk breast cancer patients, including three well-conducted randomized controlled trials (11-14). Between 1982 and 1989, the DBCG 82b trial enrolled 1,708 high-risk premenopausal patients with breast cancer after modified radical mastectomy (12). The patients were randomized to receive 


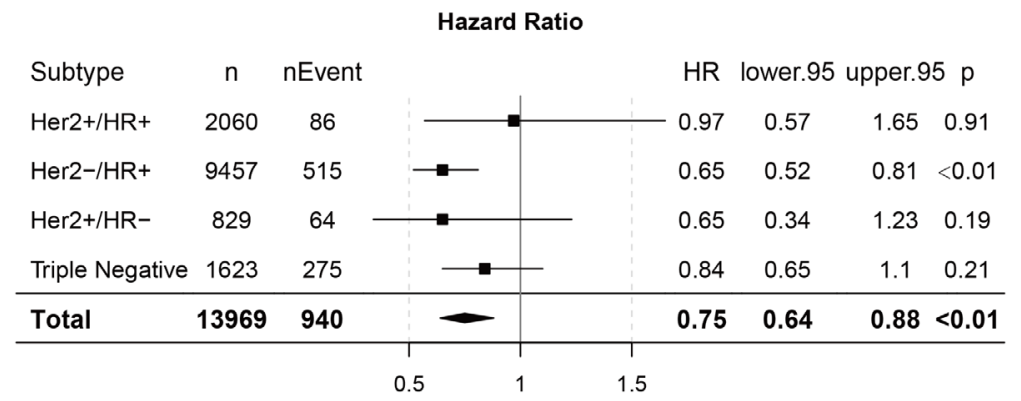

Figure 7 Forest plot of OS based on molecular subtypes. The hazard ratio (HR) was calculated by multivariate Cox proportional hazard model adjusted for radiation, race, diagnosis year, age group, surgery and chemotherapy. LN, lymph node. The data source was the LN1-3 and $\mathrm{T} 1,2$ subgroup.

cyclophosphamide-methotrexate-fluorouracil (CMF) chemotherapy alone or chemotherapy with PMRT to the chest wall and regional lymph nodes. With a 114-month median follow-up, the chemotherapy with PMRT group demonstrated a statistically significant reduction in LRR (9\% vs. $32 \%, \mathrm{P}<0.001)$ and improvement in OS $(54 \%$ vs. $45 \%, \mathrm{P}<0.001)$. Between 1982 and 1989, the DBCG 82c trial randomized 1,375 postmenopausal high-risk patients after modified radical mastectomy with a one-year course of tamoxifen or with concurrent PMRT to the chest wall and regional lymph nodes. With a 10 -year median follow-up, the tamoxifen and PMRT groups demonstrated a statistically significant reduction in LRR ( $8 \%$ vs. $35 \%, \mathrm{P}<0.001)$ and improved OS (45\% vs. 36\%, $\mathrm{P}=0.03)$. The British Columbia Trial (14) enrolled 318 node-positive premenopausal patients with breast cancer between 1978 and 1986. All subjects were randomized to CMF chemotherapy alone or CMF chemotherapy with PRMT to the chest wall and regional lymph nodes after modified radical mastectomy. After a 15-year median follow-up, the CMF chemotherapy and PRMT group demonstrated statistically significant lower LRR (13\% vs. 33\%, $\mathrm{P}=0.003)$ and improved OS (54\% vs. $46 \%, \mathrm{P}=0.07)$. The 20 -year result was consistent with the previous report showing that the CMF chemotherapy and PRMT group had statistically significantly improved survival free of isolated locoregional disease $(90 \%$ vs. $74 \%$, $\mathrm{P}=0.002)$ and improved $\mathrm{OS}(47 \%$ vs. $37 \%, \mathrm{P}=0.03)(11)$. These studies and the following EBCTCG meta-analysis demonstrated that PMRT not only decreased the risk of LRR but also improved survival for patients with high-risk breast cancer (15). Our results were consistent with those of the previous studies. Because the PMRT group contained more high-risk patients, it demonstrated inferior cumulative six-year survival. However, after controlling for potential confounders, PMRT can significantly improve BCSS and OS. Interestingly, the HR for OS did not differ significantly between these studies, even with decades of improvement in diagnosis and treatment. This indicates that in the era of modern adjuvant therapy algorithms, such as anthracycline and/or taxane-based chemotherapy, targeted therapy, and modern endocrine therapy, PMRT can still confer a similar survival benefit for breast cancer patients.

Whether patients with 1-3 positive lymph nodes can benefit from PMRT continues to be controversial. Some studies support PMRT for patients with 1-3 positive lymph nodes $(7,8,12)$; however, other studies have come to the opposite conclusion $(11,14,16)$. These inconsistent results may be due to disparities in criteria for patient selection, different therapeutic strategies, the small number of patients involved, and lack of some important prognostic factors such as ER, PR, and HER2 statuses. We conducted a population-based study utilizing the SEER 18 registries custom database, which included 77,587 breast cancer patients with 1-3 positive lymph nodes. This large cohort can provide more comprehensive results for application in real-world situations. Furthermore, all these patients were treated between 2010 and 2015, which reflected the results of state-of-the-art treatment algorithms. Additionally, this study contained some important prognostic factors, such as ER, PR, and HER2 statuses, and excluded relevant confounders compared with the studies described above. In the multivariate Cox proportional hazard regression model, the PMRT group demonstrated significantly improved BCSS and OS for patients with 1-3 positive axillary lymph nodes irrespective of $\mathrm{T}$ stage.

Because T3 and T4 patients have a high risk of LRR 
and can benefit from radiation therapy, patients with 1-3 positive axillary lymph nodes may benefit from radiation therapy because they have T3-4 tumors. Patients with 1-3 positive axillary lymph nodes and T1-2 tumors were defined as "intermediate risk" and demonstrated LRR rates of $6 \%$ to $13 \%$ after mastectomy and chemotherapy (17-19). Whether radiotherapy can reduce the LRR of such patients is still controversial $(18,20-24)$. Some studies have suggested that PMRT can reduce the LRR of such patients $(18,22)$, while others have suggested the opposite $(20,23)$. McBride et al. (21) reported that PMRT could reduce LRR for patients in the early era, whereas PMRT did not reduce LRR for patients in the later era (21). This may be due to advances in other adjuvant treatment techniques leading to decreased LRR; for patients who had not received radiation, patients of the later era had lower LRR than those of the early era. Yang et al. (24) reported that PMRT did not reduce LRR for such patients but did for patients with high recurrence factors. For example, for ER-negative patients with positive lymphovascular invasion, PMRT reduced LRR (from $40 \%$ to $12.5 \%$ ) and at the same time improved five-year OS (from $43.7 \%$ to $87.1 \%$ ). Smith et al. (20) suggested that PMRT conferred a $15 \%$ to $20 \%$ relative reduction in mortality for patients with seven or more positive axillary lymph nodes and T1-2 tumors but not for patients with fewer than seven positive axillary lymph nodes and T1-2 tumors. In the present study, the PMRT group demonstrated both improved BCSS and OS in patients with 1-3 positive axillary lymph nodes and $\mathrm{T} 1-2$ tumors. Additionally, in the subgroup analysis stratified according to $\mathrm{T}$ stage, the PMRT group demonstrated significantly improved BCSS and OS for patients at all T stages, except T1. Similarly, Fodor et al. (16) demonstrated that for patients with $1-3$ positive axillary lymph nodes, PMRT can significantly reduce LRR ( $8 \%$ vs. $24 \%, \mathrm{P}=0.01$ ) in the T2 subgroup, whereas the PMRT and no-PMRT groups demonstrated similar rates of LRR ( $8 \%$ vs. $8 \%, \mathrm{P}=0.9$ ) in the T1 subgroup. Some other studies identified T2 as an indicator of higher LRR for patients with 1-3 positive lymph nodes without PMRT (16,25-27). These patients with high risk of LRR may benefit from PMRT. Given the data above, PMRT may be applied to the T2 subgroup but not the T1 subgroup with 1-3 positive axillary lymph nodes.

Breast cancer is heterogeneous. Based on the intrinsic molecular portraits, breast cancer can be divided into four intrinsic biologic subtypes (luminal A, luminal B, HER2enriched, and triple-negative). Each subtype presents with different biologic behavior, response to treatment, and prognosis $(28,29)$. Some studies have demonstrated that the risk of LRR was different for the four intrinsic biologic subtypes. Abdulkarim et al. (30) reported that the LRR in patients with T1-2N0 triple-negative breast cancer treated with modified radical mastectomy and without PMRT was $10 \%$ at a median follow-up of 7.2 years. Voduc et al. (31) analyzed the risk of LRR for each breast cancer molecular subtype. The 10-year local relapse-free survival and regional relapse-free survival for the luminal A subtype were $92 \%$ and $96 \%$, respectively. The corresponding rates were $86 \%$ and $88 \%$ for the luminal B subtype, $83 \%$ and $88 \%$ for the HER2-enriched subtype, and $81 \%$ and $80 \%$ for the basal subtype. Dominici et al. (32) examined the risk of LRR for 819 patients after mastectomy without PMRT. The five-year risk of LRR was $2.5 \%$ for the entire cohort, $1 \%$ for the HR+/HER2- subtype, $6.5 \%$ for the HR+/HER2+ subtype, $2 \%$ for the HR-/HER $2+$ subtype, and $10.9 \%$ for the HR-/HER2 - subtype $(\mathrm{P}<0.01)$. Billar et al. (33) retrospectively reviewed the recurrence rate of 1,061 patients, of which $35 \%$ received mastectomy. The triplenegative patients demonstrated significantly higher LRR (5.7\% for the triple-negative subtype, $2.9 \%$ for the HER2+ subtype, and $1.0 \%$ for $\mathrm{ER}+$ patients, $\mathrm{P}=0.001$ ). These data suggest that the triple-negative subtype is more aggressive and demonstrated the highest rate of LRR.

The responses of different subtypes to PMRT may be heterogeneous. Patients with high risk of local recurrence may not benefit from PMRT. Kyndi et al. (34) analyzed 1,000 of the 3,083 high-risk breast cancer patients randomized to systemic therapy alone or systemic therapy and PMRT in the DBCG $82 \mathrm{~b}$ and c trials. All patients were categorized into four subtypes: ER+/HER2-, ER+/HER2+, ER-/HER2-, and ER-/HER2+. PMRT decreased LRR significantly in all subtypes, except the ER-/HER2+ subtype; however, PMRT only improved OS significantly in the ER+/HER2- subtype. Some studies have found inconsistent conclusions about whether early triple-negative breast cancer can benefit from PMRT. Wang et al. (35) conducted a randomized trial in China; 681 consecutive patients with triple-negative stage I-II breast cancer who received mastectomy were randomized to systemic chemotherapy alone or PMRT after systemic chemotherapy. All patients were stage T1 or T2, and over $80 \%$ were node-negative. PMRT significantly improved both the five-year recurrence-free survival (HR, 0.77; 95\% CI, 0.72-0.98; $\mathrm{P}=0.02$ ) and the five-year OS (HR, $0.79 ; 95 \%$ CI, 0.74-0.97; $\mathrm{P}=0.03)$. The inconsistent results may be due to disparities in patient selection criteria, 
treatment algorithms, and follow-up periods. Additionally, in the Kyndi et al. study (34), the patient numbers of some subtypes were limited in their power to detect differences. However, studies providing data about whether patients with 1-3 positive axillary lymph nodes and T1-2 tumors of different molecular subtypes can benefit from PRMT are scarce. In the present study, we selected 13,969 patients with 1-3 positive axillary lymph nodes and T1-2 tumors and conducted a subgroup analysis stratified according to molecular subtypes. The results indicated that PMRT only conferred survival benefits to the HER2-/HR+ subgroup, which was consistent with the findings of Kyndi et al. (34). This conclusion is contrary to the traditional view that subinvasive subtypes are more likely to benefit from PMRT. This may be because the benefit of radiotherapy for patients is mainly the provision of local control, and the death of patients is mainly related to distant metastasis because subtypes other than the HER2-/HR+ subtype have greater distant metastatic potential.

The advantage of this study is the large sample size, with enough samples in each subgroup to produce sufficient statistical power. Our study has some limitations. First, as declared by the Data-Use Agreement for SEER Radiation Therapy and Chemotherapy Information, the overall sensitivity was $80 \%$ for all SEER radiation therapy data, and the specificity was high; radiotherapy data are classified by the type of RT received or no/unknown. However, underreporting of PMRT use makes it more difficult to obtain positive results for this study; thus, the positive results obtained in this study are more reliable. Second, some treatment information, such as endocrine and targeted therapies, is not available in the SEER 18 registries custom database. Last, some clinicopathological factors, such as lymphovascular invasion and $\mathrm{Ki}-67$, were not available. These limitations may confer some bias to this study.

In conclusion, even in the era of modern therapy, PMRT can confer survival benefits to breast cancer patients with 1-3 positive axillary lymph nodes. Furthermore, for patients with 1-3 positive axillary lymph nodes and T1-2 tumors, PMRT can provide survival benefits. Additional randomized controlled clinical trials are needed to further support this conclusion.

\section{Acknowledgments}

We want to thank Dr. Yang Cao for his support in data analysis.

Funding: None.

\section{Footnote}

Reporting Checklist: The authors have completed the STROBE reporting checklist. Available at http://dx.doi. org/10.21037/tcr-20-3337

Conflicts of Interest: All authors have completed the ICMJE uniform disclosure form (available at http://dx.doi. org/10.21037/tcr-20-3337). The authors have no conflicts of interest to declare.

Ethical Statement: The authors are accountable for all aspects of the work in ensuring that questions related to the accuracy or integrity of any part of the work are appropriately investigated and resolved. The study was conducted in accordance with the Declaration of Helsinki (as revised in 2013). This article does not contain any studies with human participants or animals performed by any of the authors. Informed consent was not required because this was a population-based retrospective study, and no personal information was not involved.

Open Access Statement: This is an Open Access article distributed in accordance with the Creative Commons Attribution-NonCommercial-NoDerivs 4.0 International License (CC BY-NC-ND 4.0), which permits the noncommercial replication and distribution of the article with the strict proviso that no changes or edits are made and the original work is properly cited (including links to both the formal publication through the relevant DOI and the license). See: https://creativecommons.org/licenses/by-nc-nd/4.0/.

\section{References}

1. Kurtz J. The curative role of radiotherapy in the treatment of operable breast cancer. Eur J Cancer 2002;38:1961-74.

2. Truong PT, Olivotto IA, Whelan TJ, et al. Clinical practice guidelines for the care and treatment of breast cancer: 16.Locoregional post-mastectomy radiotherapy. CMAJ 2004;170:1263-73.

3. Sautter-Bihl ML, Souchon R, Budach W, et al. DEGRO practical guidelines for radiotherapy of breast cancer II. Postmastectomy radiotherapy, irradiation of regional lymphatics, and treatment of locally advanced disease. Strahlenther Onkol 2008;184:347-53.

4. Taylor ME, Haffty BG, Rabinovitch R, et al. ACR appropriateness criteria on postmastectomy radiotherapy expert panel on radiation oncology-breast. Int J Radiat 
Oncol Biol Phys 2009;73:997-1002.

5. Goldhirsch A, Winer EP, Coates AS, et al. Personalizing the treatment of women with early breast cancer: highlights of the St Gallen International Expert Consensus on the Primary Therapy of Early Breast Cancer 2013. Ann Oncol 2013;24:2206-23.

6. Kunkler IH, Canney P, van Tienhoven G, et al. Elucidating the role of chest wall irradiation in 'intermediate-risk' breast cancer: the MRC/EORTC SUPREMO trial. Clin Oncol (R Coll Radiol) 2008;20:31-4.

7. Overgaard M, Nielsen HM, Overgaard J. Is the benefit of postmastectomy irradiation limited to patients with four or more positive nodes, as recommended in international consensus reports? A subgroup analysis of the DBCG 82 b\&c randomized trials. Radiother Oncol 2007;82:247-53.

8. McGale P, Taylor C, Correa C, et al. Effect of radiotherapy after mastectomy and axillary surgery on 10-yearrecurrence and 20-year breast cancer mortality: meta-analysis of individual patient data for 8135 women in 22 randomised trials. Lancet 2014;383:2127-35.

9. Cheng SH, Horng CF, West M, et al. Genomic prediction of locoregional recurrence after mastectomy in breast cancer. J Clin Oncol 2006;24:4594-602.

10. Greenbaum MP, Strom EA, Allen PK, et al. Low locoregional recurrence rates in patients treated after 2000 with doxorubicin based chemotherapy, modified radical mastectomy, and post-mastectomy radiation. Radi-other Oncol 2010;95:312-6.

11. Ragaz J, Olivotto IA, Spinelli JJ, et al. Locoregional radiation therapy in patients with high-risk breast cancer receiving adjuvant chemotherapy: 20-year results of the British Columbia randomized trial. J Natl Cancer Inst 2005;97:116-26.

12. Overgaard M, Hansen PS, Overgaard J, et al. Postoperative radiotherapy in high-risk premenopausal women with breast cancer who receive adjuvant chemotherapy. Danish Breast Cancer Cooperative Group 82bTrial. N Engl J Med 1997;337:949-55.

13. Overgaard M, Jensen MB, Overgaard J, et al. Postoperative radiotherapy in high-risk postmenopausal breast-cancer patients given adjuvant tamoxifen: Danish Breast Cancer Cooperative Group DBCG 82crandomised trial. Lancet 1999;353:1641-8.

14. Ragaz J, Jackson SM, Le N, et al. Adjuvant radiotherapy and chemotherapy in node-positive premenopausal women with breast cancer. N Engl J Med 1997;337:956-62.

15. Clarke M, Collins R, Darby S, et al. Effects of radiotherapy and of differences in the extent of surgery for early breast cancer on local recurrence and 15-year survival: an overview of the randomised trials. Lancet 2005;366:2087-106.

16. Fodor J, Polgar C, Major T, et al. Locoregional failure 15 years after mastectomy in women with one to three positive axillary nodes with or without irradiation the significance of tumor size. Strahlenther Onkol 2003;179:197-202.

17. Katz A, Strom EA, Buchholz TA, et al. Locoregional recurrence patterns after mastectomy and doxorubicin-based chemotherapy: implications for postoperative irradiation. J Clin Oncol 2000;18:2817-27.

18. Woodward WA, Strom EA, Tucker SL, et al. Locoregional recurrence after doxorubicin-based chemotherapy and postmastectomy: Implications for breast cancer patients with early-stage disease and predictors for recurrence after postmastectomy radiation. Int J Radiat Oncol Biol Phys 2003;57:336-44.

19. Recht A, Gray R, Davidson NE, et al. Locoregional failure 10 years after mastectomy and adjuvant chemotherapy with or without tamoxifen without irradiation: experience of the Eastern Cooperative Oncology Group. J Clin Oncol 1999;17:1689-700.

20. Smith BD, Smith GL, Haffty BG. Postmastectomy radiation and mortality in women with T1-2 node-positive breast cancer. J Clin Oncol 2005;23:1409-19.

21. McBride A, Allen P, Woodward W, et al. Locoregional recurrence risk for patients with T1,2 breast cancer with 1-3 positive lymph nodes treated with mastectomy and systemic treatment. Int J Radiat Oncol Biol Phys 2014;89:392-8.

22. Tendulkar RD, Rehman S, Shukla ME, et al. Impact of postmastectomy radiation on locoregional recurrence in breast cancer patients with 1-3 positive lymph nodes treated with modern systemic therapy. Int J Radiat Oncol Biol Phys 2012;83:e577-81.

23. Moo TA, McMillan R, Lee M, et al. Selection criteria for postmastectomy radiotherapy in $\mathrm{t} 1-\mathrm{t} 2$ tumors with 1 to 3 positive lymph nodes. Ann Surg Oncol 2013;20:3169-74.

24. Yang PS, Chen CM, Liu MC, et al. Radiotherapy can decrease locoregional recurrence and increase survival in mastectomy patients with $\mathrm{T} 1$ to $\mathrm{T} 2$ breast cancer and one to three positive nodes with negative estrogen receptor and positive lymphovascular invasion status. Int J Radiat Oncol Biol Phys 2010;77:516-22.

25. Wallgren A, Bonetti M, Gelber RD, et al. Risk factors for locoregional recurrence among breast cancer patients: results from International Breast Cancer Study Group Trials I through VII. J Clin Oncol 2003;21:1205-13. 
26. Taghian A, Jeong JH, Mamounas E, et al. Patterns of locoregional failure in patients with operable breast cancer treated by mastectomy and adjuvant chemotherapy with or without tamoxifen and without radio-therapy: results from five National Surgical Adjuvant Breast and Bowel Project randomized clinical trials. J Clin Oncol 2004;22:4247-54.

27. Truong PT, Olivotto IA, Kader HA, et al. Selecting breast cancer patients with T1-T2 tumors and one to three positive axillary nodes at high postmastectomy locoregional recurrence risk for adjuvant radiotherapy. Int J Radiat Oncol Biol Phys 2005;61:1337-47.

28. Perou CM, Sorlie T, Eisen MB, et al. Molecular portraits of human breast tumours. Nature 2000;406:747-52.

29. Cancer Genome Atlas Network. Comprehensive molecular portraits of human breast tumours. Nature 2012;490:61-70.

30. Abdulkarim BS, Cuartero J, Hanson J, et al. Increased risk of locoregional recurrence for women with T12N0 triple-negative breast cancer treated with modified radical mastectomy without adjuvant radiation therapy compared with breast-conserving therapy. J Clin Oncol

Cite this article as: Yang J, Zhang X, Ye Y, Lin Y, Yang Q, Cai H. Postmastectomy radiation therapy can improve survival for breast cancer patients with 1-3 positive axillary lymph nodes: a retrospective cohort study using the SEER database. Transl Cancer Res 2021;10(5):1984-2001. doi: 10.21037/tcr-20-3337
2011;29:2852-8.

31. Voduc KD, Cheang MC, Tyldesley S, et al. Breast cancer subtypes and the risk of local and regional relapse. J Clin Oncol 2010;28:1684-91.

32. Dominici LS, Mittendorf EA, Wang X, et al. Implications of constructed biologic subtype and its relationship to locoregional recurrence following mastectomy. Breast Cancer Res 2012;14:R82.

33. Billar JA, Dueck AC, Stucky CC, et al. Triple-negative breast cancers: unique clinical presentations and outcomes. Ann Surg Oncol 2010;17 Suppl 3:384-90.

34. Kyndi M, Sorensen FB, Knudsen H, et al. Estrogen receptor, progesterone receptor, HER-2, and response to postmastectomy radiotherapy in high-risk breast cancer: the Danish Breast Cancer Cooperative Group. J Clin Oncol 2008;26:1419-26.

35. Wang J, Shi M, Ling R, et al. Adjuvant chemotherapy and radiotherapy in triple-negative breast carcinoma: a prospective randomized controlled multi-center trial. Radiother Oncol 2011;100:200-4. 


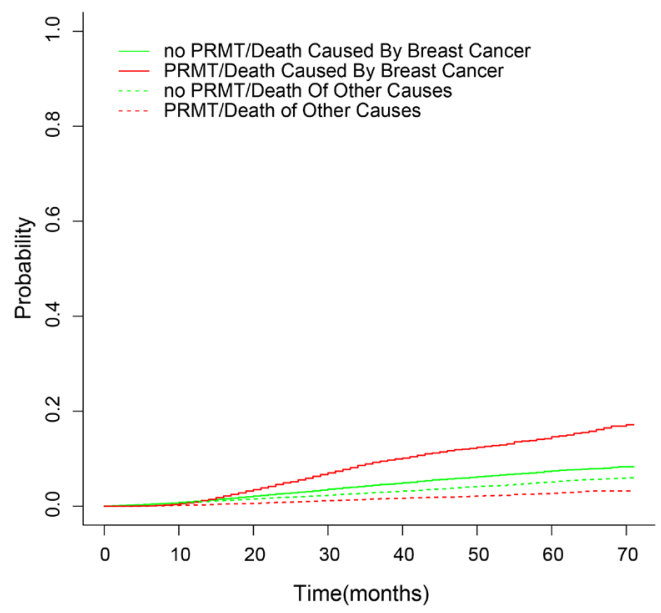

Figure S1 The cumulative incidence probability based on competing risk model. The death attributed to other causes were grouped together, and treated as the alternative event in the model. 